\title{
Perfil do uso de Medicamentos em Pacientes Autistas Acompanhados na APAE de um Município do Interior da Bahia
}

\author{
Lívia Fernandes ${ }^{l}$; Fernanda Santos Portela ${ }^{2}$; \\ Pablo Maciel Brasil Moreira ${ }^{3}$; Mauro Teles Fernandes ${ }^{4}$
}

\begin{abstract}
Resumo: O autismo é um distúrbio neurológico, na qual, os portadores apresentam em comum desordens típicas, como, dificuldade de interação social e na comunicação, além de exibir movimentos com práticas repetitivas e peculiares. Este transtorno é considerado um problema de saúde púbica e geralmente, outros problemas neuronais estão associadas, proporcionando assim, um agravo na qualidade de vida do portador. Dessa forma, o presente trabalho propõe avaliar o perfil do uso de medicamentos em pacientes autistas acompanhados na APAE de um município do interior da Bahia. Metodologia: O trabalho propõe uma delineamento descritivo exploratório de proporção qualitativo e quantitativo. O local do estudo escolhido para elaboração deste projeto foi a Associação de Pais e Amigos dos Excepcionais - APAE do município de Vitória da Conquista, localizado no interior da Bahia. O trabalho contou com uma população de crianças autistas, com idades entre 03-14 anos, de ambos os sexos. O instrumento para coleta de dados se equivale a um roteiro na qual, algumas questões foram levantadas para elaboração do projeto. Os dados foram tabulados pelos programas Microsoft Excel® e Microsoft Word® do ano 2013. A análise dos questionários ocorreu de maneira individual, pois ocorreram algumas observações peculiares que não tinham sido perguntadas no momento da entrevista, mas a genitora informou. Resultados: De acordo com a análise dos dados, os resultados encontrados foram bastante condizentes com a literatura e evidenciaram uma maior prevalência em pacientes autistas do gênero masculino com $86 \%$ dos pacientes escolhidos, e no que diz respeito a faixa etária, $46 \%$ possuíam entre seis a 11 anos, além disso, o tempo de diagnóstico foi condizente a este seguimento, pois $72 \%$ dos pacientes, foram diagnosticados com idades entre dois a cinco anos. Dos pacientes participantes, 58\% não apresentavam patologias associadas à síndrome e 96\% utilizam medicamento, na qual, o mais comum foi o risperidona com $41 \%$. Dos $96 \%$ que administram a terapia medicamentosa, $60 \%$ apresentam entre dois a cinco anos de tratamento, $35 \%$ relatam como efeito indesejável a sonolência e $75 \%$ recebem medicamento diretamente da genitora, na qual, $98 \%$ das responsáveis sabem da importância de manter a terapia correta. Conclusão: pode-se concluir que o trabalho obteve resultados esperados e satisfatórios frente ao perfil dos pacientes cadastrados na APAE do município. No entanto o presente local do estudo não possui a presença do farmacêutico que é o profissional habilitado a promover o uso racional de medicamentos diminuindo assim os riscos de erros e reações adversas, o mesmo deve estar inserido na equipe multiprofissional buscando oferecer melhorias na farmacoterapia contribuindo para evolução clínica dos pacientes e sua qualidade de vida.
\end{abstract}

Descritores: Assistência Farmacêutica. Autismo infantil. Medicamento.

\section{Medications Profile Use in Autistic Patients Accompanied in the APAE in a Municipality of Bahia}

\begin{abstract}
Autism is a neurological disorder, in which the carriers present in common typical disorders, such as difficulty in social interaction and communication, in addition to displaying movements with repetitive and peculiar practices. This disorder is considered a public health problem and generally, other neural problems are associated, thus providing, an impairment in the quality of life of the wearer. Thus, the present study proposes to evaluate the profile of the use of drugs in autistic patients accompanied in the APAE of a city in the interior of Bahia. Methodology: This work proposes a descriptive exploratory delineation of qualitative and quantitative proportions. The study site chosen for this project was the Association of Parents and Friends of the Exceptional APAE of the municipality of Vitória da Conquista, located in the interior of Bahia. The study had a population of
\end{abstract}

\footnotetext{
${ }^{1}$ Graduanda do Curso de Farmácia da Faculdade Independente do Nordeste (FAINOR). E-mail:liviafernandes0903@ gmail.com

${ }^{2}$ Graduada em Farmácia pela Faculdade Independe do Nordeste (FAINOR), Pós-graduada em análises clínicas e toxicologia pela FAINOR, atualmente atua como farmacêutica em uma farmácia privada. E-mail: fernandaportela@yahoo.com.br

${ }^{3}$ Farmacêutico na Secretaria Municipal de Saúde de Vitória da Conquista - Bahia; Especialista em Gestão da Assistência Farmacêutica pela Universidade Federal de Santa Catarina. E-mail: pablomaciel.farmacia@gmail.com

${ }^{4}$ Graduado em Farmácia pela Universidade Federal da Bahia (UFBA), Docente da Faculdade Independente do Nordeste -

FAINOR, Vitória da Conquista/BA. Mestre em Saúde Pública - ENSP-FIOCRUZ. E-mail: maurofteles@ hotmail.com
} 
autistic children, aged between 03-14 years, of both sexes. The instrument for data collection is equivalent to a roadmap in which, some questions were raised to elaborate the project. The data were tabulated by the Microsoft Excel ${ }^{\circledR}$ and Microsoft Word ${ }^{\circledR}$ programs of the year 2013. The analysis of the questionnaires occurred individually, as there were some peculiar observations that had not been asked at the time of the interview, but the giver reported. Results: According to the data analysis, the results found were quite consistent with a literature and showed a higher prevalence in male patients with $86 \%$ of the selected patients and $46 \%$ had between six and 11 years, besides the time Was consistent with this follow-up, and $72 \%$ of the patients were diagnosed with two people in five years. Participating doses, 58\% had no pathology associated with the syndrome and $96 \%$ used medication in quality, or more common for risperidone with $41 \%$. Of the $96 \%$ who administer the drug therapy, $60 \%$ are treated with five years of treatment, $35 \%$ report as undesirable effect with drowsiness and $75 \%$ receive the medicine directly from the mother, in quality, $98 \%$ correct therapy. Conclusion: It can be concluded that the study obtained expected and satisfactory results in relation to the profile of the patients enrolled in the APAE of the municipality. However, the present study site does not have the presence of the pharmacist who is the professional qualified to promote the rational use of medicines, thus reducing the risks of errors and adverse reactions, the same must be inserted in the multiprofessional team seeking to offer improvements in pharmacotherapy contributing to Clinical evolution of patients and their quality of life.

Keywords: Pharmaceutical Assistance. Childhood autism. Medication.

\section{Introdução}

Os transtornos neurológicos acometem muitos indivíduos e estas condições clínicas, geralmente, são de origem multifatoriais. Haja vista, o indivíduo acometido por algum transtorno pode apresentar uma predisposição genética ou adquiri-lo através de um fator desencadeante ao longo do tempo, ou ainda, através da combinação das duas variáveis citadas, dentre outros. Nessa instância, a gama dos distúrbios mentais é extensa e como exemplo dessas desordens, encontra-se o transtorno autista (PEREIRA, 2008; HALPERN; FIGUEIRAS, 2004).

O distúrbio autista é atualmente classificado como Transtorno do Espectro Autista (TEA). A TEA é uma classe descrita, para abranger alguns transtornos, dentre os quais, os portadores apresentam em comum desordens típicas, como, dificuldade de interação social e na comunicação, além de exibir movimentos com práticas repetitivas e peculiares. O termo autismo é considerado uma síndrome, pois não existe um consenso bem definido sobre quais as causas que proporcionam a origem deste transtorno, pois, pode ter um seguimento genético, porém, acredita-se que este não seja o único fator para o desenvolvimento (BRITO; VANCONCELOS, 2016; MEIMES; SALDANHA; BOSA, 2015; GARCIA; et al., 2016; SEMENSATO; BOSA, 2014; SILVA; FRIGHETTO; SANTOS, 2013; SILVA; CHAVES, 2014).

Neste contexto, estima-se que aproximadamente 500 mil pessoas, no Brasil, sofram com o autismo. Em média, uma entre 66 crianças, é diagnosticada com a síndrome. Assim, este transtorno é considerado um problema de saúde púbica e geralmente, outros problemas neuronais estão associadas, como o retardo mental e quadros de crises convulsivas, proporcionando assim, um agravo na qualidade 
de vida do portador (LEAL; et al., 2015; BECKER; et al., 2016; MAIA FILHO; et al., 2016; COUTO; DUARTE; DELGADO, 2008; SOUZA; et al., 2008).

A convulsão é uma desordem da atividade cerebral expressada de maneira exacerbada ou descoordenada, o que ocasiona sérios riscos ao paciente. (DURANTE; CANCELIER, 2007; FIGUEIRÓ-FILHO; et al., 2004). As crises convulsivas estão frequentemente associadas ao distúrbio do autismo, e se propagam em aproximadamente $7 \%$ das crianças, em idade pré-escolar (PEREIRA; RIESGO; WAGNER, 2008; MATTOS; PONDÉ; CALMON, 2016).

O retardo mental, por sua vez, é a condição patológica que afeta a porção neuronal do indivíduo e compromete sua capacidade de desenvolvimento e discernimento de certas atividades e comportamentos (VASCONCELLOS, 2004). Este agravo também é muito comum em crianças autistas e o perfil estimado se estende de $65 \%$ até $90 \%$ dos portadores autistas (CAMARGO; BOSA, 2009)

Diante destas informações expostas que afetam muitas crianças autistas, a ampla farmacoterapia, na maioria das vezes é necessária. Sendo imposta, habitualmente, como tratamento inicial para o paciente (OLIVEIRA; et al., 2015). O acompanhamento farmacoterapêutico se faz essencial pois, os portadores são, geralmente, crianças, e as genitoras ou responsáveis administram os medicamentos o que pode interferir na adesão terapêutica do paciente (NIKOLOV; JONKER; SCAHILL, 2006; OLIVEIRA; FERREIRA, 2010).

Contudo, o profissional mais apto à realização e condução de um seguimento farmacoterapêutico, é o farmacêutico. Essa escolha se deve ao fato deste profissional conhecer sobre medicamentos e suas interações, além de sugerir uma melhor conduta no manejo terapêutico e evitar problemas relacionados ao uso dos medicamentos (SCARCELA; MUNIZ; CIRQUEIRA, 2011).

A relevância desse estudo se deve ao fato de não haver muitos registros na literatura, sobre este tema, além de não possuírem trabalhos atuais envolvidos com crianças portadoras de problemas mentais, deste espectro, no município. Visando este fim, houve uma necessidade de desenvolver este trabalho. Dessa forma, o presente trabalho propõe avaliar a importância do acompanhamento farmacoterapêutico em crianças autistas em um município do interior da Bahia.

\section{Metodologia}

O trabalho possui um delineamento descritivo e exploratório de caráter quantitativo. A pesquisa descritiva baseia-se na descrição de acontecimentos pertinentes à observação, descrição e classificação dos acontecimentos analisados de uma população e suas variáveis, e pode-se dividir em pesquisa 
bibliográfica e de campo. Em relação a natureza da pesquisa, o termo quantitativo propõe uma quantificação das informações obtidas pelos entrevistados (GIL, 2002; DIEHL, 2004).

O local do estudo escolhido para elaboração deste projeto foi a Associação de Pais e Amigos dos Excepcionais - APAE do município de Vitória da Conquista, localizado no interior da Bahia. Esta cidade possui aproximadamente 346.069 habitantes e mantém uma distância de $509 \mathrm{~km}$ da capital Salvador (IBGE, 2016).

A APAE é uma entidade filantrópica e se volta para proporcionar uma melhoria na qualidade e perspectiva de vida, por indivíduos que possuam alguma deficiência ou alteração neurológica que as impeçam de manter um convívio social com outros indivíduos (FENAPAES, 2003).

O trabalho utilizou uma amostra composta por 50 pacientes da APAE do município, com idades entre 03-14 anos, de ambos os sexos e escolhidas aleatoriamente. Essa faixa etária foi escolhida pois em comunicação prévia com a coordenadora da instituição, ela afirmou que poucos adolescentes acima de 14 anos vão fazer o acompanhamento e acima de três anos o autismo é diagnosticado. Como fator de inclusão, as crianças que também possuíam outras condições clínicas associadas ao quadro do autismo foram escolhidas para o trabalho, enquanto que o fator de exclusão foi importante para crianças que não residam no município citado.

Os dados coletados foram extraídos através de uma entrevista com os pais ou responsáveis pelas crianças autistas, na APAE. O instrumento para coleta de dados se equivale a um roteiro na qual, algumas questões que sejam pertinentes para realização do trabalho.

O tempo médio de cada entrevista foi de 10 minutos e todas as respostas e observações que as responsáveis pelos pacientes relatavam, a autora transcrevia no questionário com bastante atenção e se houvesse necessidade, a autora pedia para solicitava repetição, a fim de evitar quaisquer dúvida e/ou troca de informações na hora de tabular os dados.

Aos que concordaram em participar deste estudo, a autora informou sobre "Termo de Consentimento Livre e Esclarecido", no qual, continham dados pertinentes aos aspectos éticos e vigentes na dispostos na Resolução no 466/12 do Conselho Nacional de Saúde (CNS), assim, a responsável pelo paciente assinava em duas vias, uma cópia para si e a outra para a autora. A entrevista ocorreu em local reservado para manter a integridade e evitar a exposição do paciente.

O projeto de pesquisa foi submetido pela Comissão de Ética em Pesquisa da Faculdade Independente do Nordeste - FAINOR e aprovado com número 6437716.0.0000.5578. Os dados foram tabulados através dos programas Microsoft Excel®, Microsoft Word® do ano 2013. 
Id on Line Revista Multidisciplinar e de Psicoloqia

Id on Line Multidisciplinary and Psycology Journal

\section{Resultados e Discussões}

Como estratégia para a coleta dos dados a serem apresentados abaixo, optou-se pela conversa direta com as pessoas que mantém vínculo direto com as crianças e que as levavam para a instituição APAE. Essa se tornou a melhor opção para compreensão e produção deste estudo, uma vez que não foi possível ter acesso inicial aos prontuários médicos dessas crianças.

Ao observar a Tabela 1, identifica-se as variáveis, características sociodemográficas, o tempo de diagnóstico para autismo e se o portador autista possuía alguma outra condição clínica associada.

Tabela 1. Características sociodemográficas, tempo de diagnóstico e associação a outra condição clínica em pacientes autistas da APAE, entre o período de Janeiro a Março de 2017.

\begin{tabular}{lrr} 
& Variáveis & \multicolumn{2}{c}{ Frequência } & \% \\
\cline { 2 - 4 } Gênero & $\mathbf{N}$ & 14,00 \\
Feminino & 7 & 86,00 \\
Masculino & 43 & \\
& & \\
Faixa Etária & & 28,00 \\
& & 46,00 \\
03 a 05 anos & 14 & 26,00 \\
06 a 11 anos & 23 & \\
11 a 15 anos & 13 &
\end{tabular}

\section{Diagnóstico de Autismo}

$\leq 1$ ano

2 a 5 anos

6 a 10 anos

$>10$ anos

\section{Condição clínica associada ao Autismo}

TDAH

Convulsão

Retardo Mental (RM)

Paralisia Cerebral (PC)

Convulsão + RM

Convulsão + RM + PC

Microcefalia

Transtornos Comportamentais e do Humor

\section{Total} 58,00

Fonte: Dados coletados pela pesquisadora em 2017. 
Dentre os resultados encontrados para a primeira variável - gênero -, o estudo aponta uma maior prevalência em pacientes autistas do gênero masculino com 86\% (43). Enquanto que apenas 14\% (7), eram representantes do sexo feminino. Estes dados versam com o trabalho de Hadjkacem e colaboradores (2016), uma vez que ao estudar 50 pacientes autistas pacientes de um Departamento Psiquiátrico, constataram que 37 eram autistas do sexo masculino.

Garcia; et al. (2016) também obtiveram resultados similares aos apurados nesta pesquisa. O percentual de autistas do sexo masculino traçado em uma escola de São Paulo/SP, foi em média 75\%. Este fato pode ser explicado por Castro; et al. (2016), pois afirmam que meninos possuem o gene SRY (sex determing region Y) no cromossomo Y. Este gene promove, entre outras funções, a regulação da monoaminoxidase A (MAO A) no Sistema Nervoso Central (SNC), assim, os portadores de autismo possuem, geralmente, um desequilíbrio dos metabólitos catalisados pela MAO A, e o fato de da presença mais forte no cromossomo Y permite que o sexo masculino seja o mais prejudicado por essa desordem.

Em relação a idade dos 50 autistas participantes da pesquisa, 46\% (23) possuíam entre seis a 11 anos, em seguida as crianças com $28 \%$ (14) apresentavam de três a cinco anos de idade. O resultado obtido foi similar à pesquisa dos autores Teixeira; Jucá (2014), pois em um Centro de Atenção Psicossocial Infanto-Juvenil de Salvador/BA maioria 53,1\% dos autistas cadastrados na instituição possuíam de três a nove anos, enquanto que 39,9\% apresentavam entre 10 e 18 anos.

De acordo com Zanon, Backes; Bosa (2014) as primeiras manifestações do transtorno aparecem ao longo dos 36 meses de vida. Segundo Silva; Frighetto; Santos (2013), nos primeiros 12 meses a criança com autismo não balbucia e não consegue fazer gestos proporcionais a esta idade. Aos 16 meses, faltam palavras e, aos 24 a mesma não consegue se comunicar com as pessoas. O diagnóstico para síndrome costuma ser aos três anos de idade, baseado nos conceitos do DSM-IV e da CID-10 (MACHADO; et al., 2014; PINTO; et al., 2016).

Em conformidade com a faixa etária dos pacientes, o tempo de diagnóstico foi condizente às informações acima, uma vez que $72 \%$ (36) dos pacientes, foram diagnosticados com idades entre dois a cinco anos. O trabalho de Silva (2015) alcançou percentual equivalente ao adquirido, pois $50 \%$ das crianças e adolescentes que estavam inseridas em uma APAE de São Paulo/SP foram diagnosticados quando tinham idades entre três a cinco anos.

Corroborando com Zanon; Backes; Bosa (2014); Gomes; et al. (2015), por ser um distúrbio neurológico crônico a família deve procurar meios de interação e introdução, da criança ou adolescente autista, na sociedade. Por isso é tão importante o diagnóstico precoce e atenção da família para com a criança, pois ajudam a melhorar o convívio e desenvolvimento emocional e na fala da criança e assim, ajudando na qualidade de vida do paciente. 
A última variável da tabela 1 expõe as condições clínicas que estão frequentemente associadas à síndrome do autismo. Dos pacientes participantes, um percentil de 58\% (29) não apresentavam patologias relacionadas à síndrome. Porém, um dado bastante relevante foi a associação do autismo com convulsão e retardo mental, dado representado por $14 \%$ (07). Segundo relato dos responsáveis entrevistados, o autismo associado somente ao retardo mental representa $8 \%$ (4) e a convulsão com o autismo teve um total de $6 \%$ (3).

Marchesan; Riesgo (2015) afirmam que os portadores do autismo possuem entre duas até cinco morbidades associadas ao quadro clínico base. Boddaert; et al. (2006) apud Fontoura (2015) estimam que as comorbidades convulsão e retardo mental estão presentes em autistas em até $70 \%$ e $33 \%$ dos casos, respectivamente. Por outrora, Silva; Mulik (2009) versam que o retardo mental é a complicação neurológica mais comum em crianças autistas e acomete aproximadamente 60 a $75 \%$ dos casos.

Pereira; Pegoraro; Cendes (2012) explicam a relação do autismo com a convulsão (e crises epilépticas), na qual, pode ser decorrente de herança genética, patologia cerebral comum ou perturbações no sistema neuronal, como o sistema límbico, por exemplo.

Os autores Machado; et al. (2014); Brito; Rosenthal; Souza (2016) entram em consenso quando determinam que o déficit e o atraso da comunicação associado a grande dificuldade de promoção ao convívio social, delimitam ao portador uma condição de retardo mental (RM). Rodrigues; Lima (2014) trazem a delimitação para o RM como restrição no funcionamento intelectual, cognitivo e comportamental da criança.

O Gráfico 1 a seguir, ilustra o percentual total dos pacientes estudados neste trabalho em relação ao uso de alguma terapia medicamentosa. De todos os $100 \%$ (50) pacientes estudados, somente $96 \%$ (48) utilizam terapia medicamentosa, portanto todas as tabelas subsequentes terão um total de 48 pacientes, devido acompanhamento por essa terapia.

Gráfico 1. Utilização de Medicamentos por pacientes autistas da APAE de um município do interior da Bahia, entre o período de Janeiro a Março de 2017.

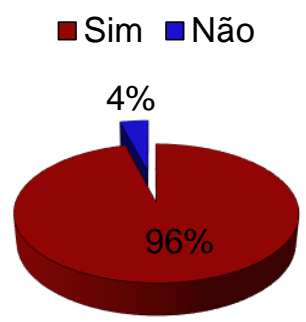

Fonte: Dados coletados pela pesquisadora em 2017. 
Como explicam Leite; Meirelles; Mihomem (2015); Amaral; et a. (2012); Oliveira; et al. (2015) a farmacoterapia pode ser indicada após o diagnóstico de autismo, a depender do grau do transtorno. Os medicamentos não tratam a síndrome, mas agem nos sintomas que frequentemente aparecem nos pacientes como, irritabilidade, agressão e perturbações comportamentais e do humor. Além disso, esta terapia é alterada de um paciente para outro, finalizando um tratamento individualizado e que possa atender outras patologias associadas ao autismo.

Garcia (2016), por sua vez, acrescenta ainda que a terapia medicamentosa em pacientes autistas não é uma conduta obrigatória. Porém, quando se torna necessária a introdução do medicamento no paciente autista, Soares; Braga (2014); Varela; Machado (2016) elucidam que o tratamento é feito, preferencialmente, com neurolépticos (ou antipsicóticos).

Em relação aos medicamentos mais utilizados pelos pacientes autistas, são demonstrados na Tabela 2 de acordo o princípio ativo.

Tabela 2. Medicamentos utilizados por pacientes autistas, com idades entre três a 14 anos, acompanhados na APAE de um município do interior da Bahia, entre o período de Janeiro a Março de 2017.

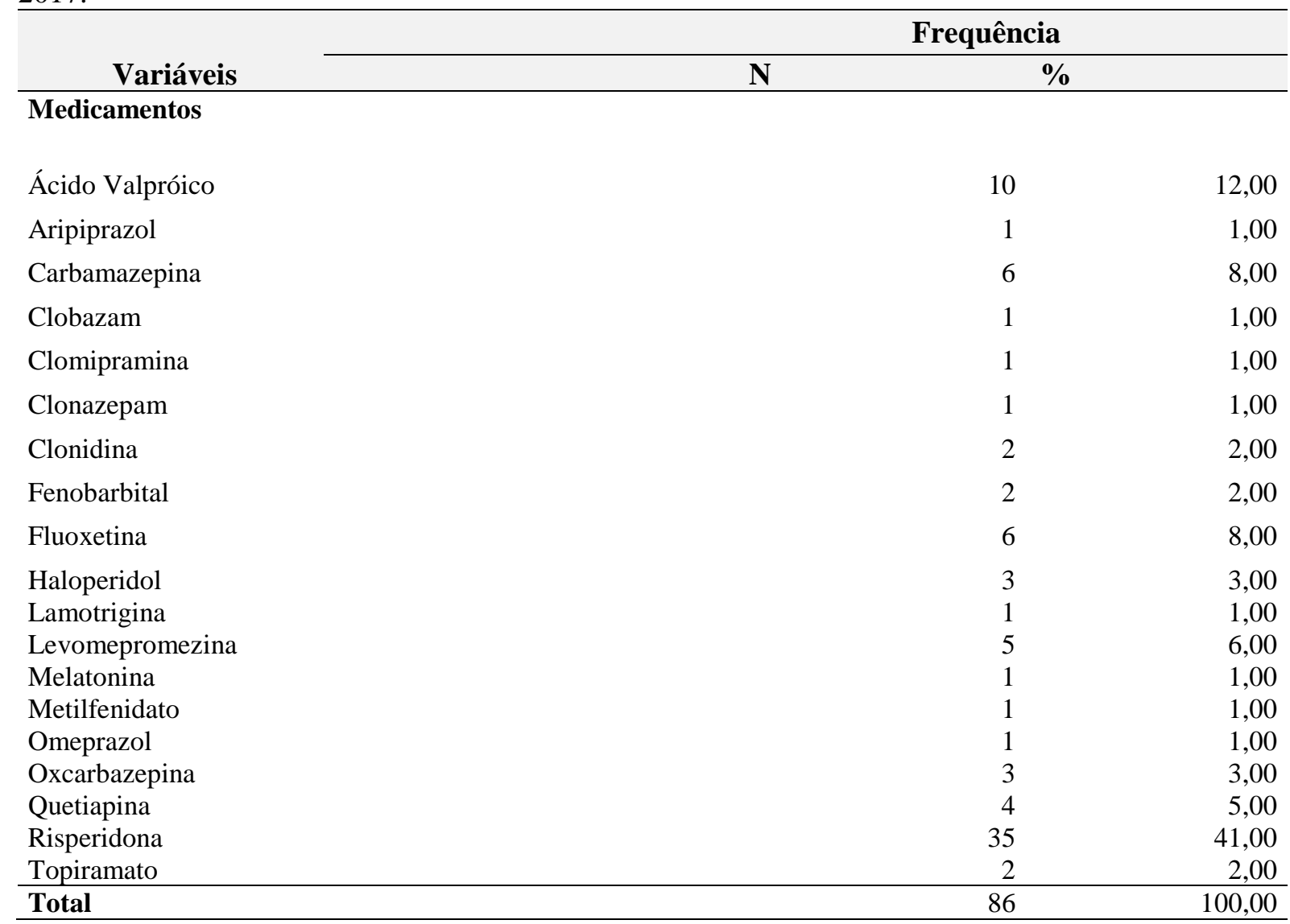

Fonte: Dados coletados pela pesquisadora em 2017. 
De acordo com os dados exibidos, o medicamento mais prescrito para o tratamento dos sintomas do autismo é risperidona $41 \%$ (35) e em seguida $12 \%$ (10) o ácido valpróico. O resultado encontrado foi relativo ao identificado por Berazain; Paz (2015), que realizaram um trabalho com 10 autistas em uma cidade na Bolívia e apenas 6 faziam uso medicamento para sintomas da síndrome, 4 portadores utilizavam risperidona e 1 seguido, utilizava ácido valpróico. Leite; Meirelles; Mihomem (2015) em seu estudo com crianças autistas no Piauí, encontrou uma porcentagem de 52\% em uso de risperidona.

Segundo Oviedo; et al. (2015) a risperidona é um medicamento classificado como antipsicótico atípico que possui mecanismo de ação, o bloqueio dos receptores do neurotransmissor, serotonina. Contudo, Binelli; Caupena; Pedreño (2013); Hervás; et al. (2012); Zampiroli; Souza (2012), acrescentam que este fármaco exerce um efeito positivo no manejo dos sintomas do autismo, principalmente, perante a irritabilidade e hiperatividade do paciente, além de não possuir tantos efeitos colaterais frente a antipsicóticos típicos.

Brandão Júnior (2009) agrega informação acerca da variedade de medicamentos para tratamento farmacológico do autismo, pois, além da intensidade dos sintomas a escolha se dá muitas vezes por "tentativa e erro", uma vez que cada paciente/ organismo vai responder de alguma forma.

A Tabela 3 adiciona informações pertinentes ao tratamento, como, o tempo da farmacoterapia e se o paciente apresenta algum evento adverso.

Tabela 3. Tempo estimado da farmacoterapia e relato de identificação dos efeitos adversos dos medicamentos utilizados por pacientes autistas, com idades entre 3 a 14 anos, acompanhados na APAE de um município do interior da Bahia, entre o período de Janeiro a Março de 2017.

\section{Frequência}

Variáveis $\mathrm{N} \quad \%$

Tempo de início da Terapia Medicamentosa

$\leq 1$ ano

2 a 5 anos

6 a 10 anos

Não sabe

\section{1}

29

7

1
23,00

60,00

15,00

2,00

\section{Efeitos Adversos aos Medicamentos}

Aumento de peso

Cefaléia

Constipação

Náuseas/ Enjoo

Perda de peso

Polifagia

5,00

Sede excessiva/ Xerostomia

5,00

Sonolência 
Id on Line Revista Multidisciplinar e de Psicoloqia

Id on Line Multidisciplinary and Psycology Journal

Tontura

Total 100,0

Fonte: Dados coletados pela pesquisadora em 2017.

A primeira variável de tempo de início da terapia medicamentosa, corrobora com o diagnóstico do autismo nos pacientes avaliados, na qual, $60 \%$ (29) apresentam entre dois a cinco anos de terapia. Como já foi elucidado acima, o tratamento medicamentoso requer disciplina e de acordo com Brzozowski; Caponi (2013); Farias; Silva; Cunha (2014), a administração de fármacos no autismo é paliativo, pois promove uma melhora no sofrimento das consequências provocadas pela condição clínica, e almeja a tentativa de "uma vida normal" para o paciente.

No que se refere ao efeito adverso provocado pelo uso dos medicamentos listados, 35\% (7) apresentam como evento indesejado a sonolência. Esta reação é muito comum em pacientes que fazem uso da risperidona e atinge cerca de 40 a 45\% dos usuários (MEDSCAPE, 2017; BRASIL, 2014). De acordo com Afonso; Viveiros; Vinhas de Souza (2011), a risperidona, provavelmente, possui um efeito indutor do sono devido ação inibitória no receptor $\alpha-1$ adrenérgico. Além deste antipsicótico, outros medicamentos também podem induzir este efeito, como por exemplo, Brito; Rosenthal; Souza (2016) citam o metilfenidato, Forsan (2010) expõe os benzodiazepínicos e os anticonvulsivantes, em que, Barreto; Massabki (2010) julgam a carbamazepina e Mano (2015), o ácido valpróico.

Por fim, a tabela 4 ressalta o cuidado para com as crianças e adolescentes autistas participantes, quanto a administração da terapia farmacológica e a importância de administrar corretamente o medicamento nos horários corretos e doses prescritas.

Tabela 4. Administração e importância da utilização da farmacoterapia em pacientes autistas, com idades entre três a 14 anos, acompanhados na APAE de um município do interior da Bahia, entre o período de Janeiro a Março de 2017.

\begin{tabular}{lrr}
\multicolumn{1}{c}{ Variáveis } & \multicolumn{2}{c}{ Frequência } \\
\cline { 2 - 4 } Quem Administra o Medicamento & N & \% \\
Mãe & 36 & 75,00 \\
Mãe e Pai & 6 & 13,00 \\
Mãe e irmã(ão) & 4 & 8,00 \\
Mãe e avó & 1 & 2,00 \\
Mãe e babá & 1 & 2,00 \\
Sabe a Importância de Administrar o & & \\
Medicamento no Paciente Autista & & \\
Sim & & \\
& 47 &
\end{tabular}


Fonte: Dados coletados pela pesquisadora em 2017.

** Obs.: A genitora só administra o medicamento no paciente quando o mesmo encontra-se agitado.

Ao analisar os resultados, nenhum paciente auto-administra o medicamento, eles precisam de auxílio até porque a maioria dos pacientes entrevistados possuem pouca idade, então, 75\% (36) recebem o cuidado da terapia medicamentosa diretamente da genitora. Por fim, dos pacientes que utilizam medicamentos, 98\% (47) das responsáveis sabem da importância de manter a terapia correta.

Em consonância com Tabaquim; et al. (2015) a presença de uma patologia crônica pode enfraquecer as ligações no seio familiar, mas Costa; et al. (2013); Ferreira; Costa; Melo (2014) denotam que o laço materno é muito forte pois planejamento e a concepção de um filho sempre foi uma grande realização para muitas famílias, então, a família pode representar responsabilidades e amadurecimentos, frente às adversidades.

Os autores Maia; et al. (2016) realizaram um estudo com pais de filhos autistas em Minas Gerais. O trabalho mostrou várias famílias de crianças autistas e explanou que os pais, principalmente a genitora, pois recebe um "choque" maior, ao idealizar um filho "normal" antes de nascer, procuram serviços para aprender mais sobre a síndrome autista e ajudar a criança a se desenvolver melhor.

\section{Considerações Finais}

Em consonância com os dados apresentados e informações da literatura expostas, pode-se concluir que o trabalho obteve resultados esperados e satisfatórios frente ao perfil dos pacientes cadastrados na APAE do município.

Quatro pontos importantes foram observados durante a coleta de dados e desenvolvimento da pesquisa. O primeiro, muitos pacientes que fazem terapia com mais de um medicamento e muitos destes fármacos podem interagir e causar um prejuízo a saúde do paciente. Haja vista, é de extrema importância que ocorra um acompanhamento farmacoterapêutico, realizada por um farmacêutico, para garantir um Uso Racional dos Medicamentos (URM), bem como garantir uma qualidade de vida melhor ao portador.

Existem poucos estudos que abordam a temática da atenção farmacêutica em pacientes autistas que praticam poli terapia. Porém, observa-se ainda que o profissional farmacêutico ainda não está totalmente inserido na equipe multiprofissional, o que reflete em uma crise de identidade profissional, deixando muitas vezes, que as pessoas e até mesmo os profissionais de saúde desconheçam o seu 
trabalho. Por isso, é importante que o farmacêutico acompanhe o paciente e notifique possíveis interações medicamentosas, pois este profissional possui conhecimento e embase suficiente para sugerir intervenções no tratamento do portador.

O segundo fator analisado foi que os portadores estudados são crianças e adolescentes e muitas vezes o efeito adverso provocado pelo medicamento atrapalha o desenvolvimento e interação social com outras pessoas. Se faz necessário, também, que a família, ou responsáveis estimulem mais as crianças, pois, durante a coleta de dados, houve uma genitora que relatou "sossego" a criança, ao administrar o medicamento e isso precisa ser desmitificado.

No ponto seguido, uma mãe relatou que só administrava o medicamento antipsicótico quando o filho sentia crises de agitação. Este tipo de medicamento é de uso contínuo e deve ser administrado em horários iguais para evitar as crises de ansiedade, comportamentais ou até mesmo psíquicas. E o último ponto, foi a administração "escondida" da família, ou seja, ainda existe um preconceito quanto ao autismo e talvez, um desconhecimento desta patologia.

\section{Referências}

AMARAL, C., O., F.; MALACRIDA, V., H.; VIDEIRAS, F., C., H.; PARIZI, A., G., S.; OLIVEIRA, A., DE; STRAIOTO, F., G. Paciente autista: métodos e estratégias de condicionamento e adaptação para o atendimento odontológico. Archives of Oral Research, v. 8 n. 2, p. 143-51, 2012

AFONSO, P.; VIVEIROS, V.; SOUSA, T., V., DE. Alterações do sono na esquizofrenia. Acta Médica Portuguesa, v. 24, s. 4, p. 799-806, 2011.

BARRETO, B., C., S.; MASSABKI, P., S. Efeitos adversos no sistema nervoso central dos fármacos antiepiléticos em idosos. Revista Brasileira de Clínica Médica, v. 8, n. 4, 2010.

BECKER, M., M.; BOSA, C.; OLIVEIRA-FREITAS, V., L.; GOLDIM, J., R.; OHLWEILER, L.; ROESLER, R.; SCHWARTSMANN, G.; RIESGO, R., DOS S. Melhoria nos sistemas do transtorno do espectro autista em três criancas, utilizando peptídeo liberador de gastrina. Jornal de Pediatria, v. 92, n. 3, p. 302-306, 2016.

BERAZAIN, C., A.; PAZ, G. Trastorno Autista en pacientes de la Caja Nacional de Salud de Cochabamba, Enero 2013 al 2015. Revista Científica Ciencia Médica, v. 18, n. 1, 2015.

BINELLI, C.; CAUPENA, G.; PEDREÑO, C. Uniendo las piezas del puzzle: a propósito de un caso de trastorno del espectro autista adulto. Revista de la Asociación Española de Neuropsiquiatría, v. 33, n. 119, 2013.

BRANDÃO JUNIOR, P., M., C. Um bebê no CAPSi: uma clínica possível. Estudos e Pesquisas em Psicologia, v. 9, n. 2, 2009.

BRASIL. Ministério da Saúde. Secretaria de Ciência, Tecnologia e Insumos Estratégicos, Departamento de Gestão e Incorporação de Tecnologias em Saúde. Risperidona no Transtorno do Espectro do Autismo (TEA). Brasília/DF, 2014.

BRITO, A., R.; VASCONCELOS, M., M., DE. conversando sobre autismo-reconhecimento precoce e possibilidades terapêuticas. In: CAMINHA, V., L.; HUGUENIN, J.; ASSIS, L., M., DE; ALVES, P., P. Autismo Vivências e Caminhos. São Paulo: Blucher, 2016. 
BRITO, T., M.; ROSENTHAL, I., A.; SOUZA, C., S., B. Avaliação do estado nutricional de pacientes atendidos em ambulatório de neuropsiquiatria infantil. International Journal of Nutrology, v. 9, n. 2, 2016.

BRZOZOWSKI, F., S.; CAPONI, S., N., C., DE. Medicalização dos Desvios de Comportamento na Infância: Aspectos Positivos e Negativos. Psicologia: ciência e profissão, v. 33, n. 1, p. 208-221, 2013.

CAMARGO, S., P., H.; BOSA, C., A. Competência social, inclusão escolar e autismo: revisão crítica da literatura. Psicologia e Sociedade, v. 21, p. 65-74, 2009.

CASTRO, C., B., DE; LIN, J.; SAKAE, T., M.; MAGAJEWSKI, F., R., L. Aspectos sociodemográicos, clínicos e familiares de pacientes com o transtorno do espectro autista no sul de Santa Catarina. Revista Brasileira de Neurologia, v. 52, n. 3, p. 20-28, 2016.

COSTA, M., S.; PATRÍCIO, C., L.; BISPO, G., M., B.; RODRIGUES, E., M., D.; DE ARAÚJO, D., B. HIV Testing: Knowledge, Meanings and Experiencesof Pregnant Women. Journal of Research Fundamental Care On Line, v. 5, n. 3, p. 10-17, 2013.

COUTO, M., C., V.; DUARTE, C., D.; DELGADO, P., G., G. A saúde mental infantil na Saúde Pública brasileira: situação atual e desafios. Revista Brasileira de Psiquiatria, v. 30, n. 4, 2008.

DIEHL, A. Pesquisa em ciências sociais aplicadas: métodos e técnicas. São Paulo: Prentice Hall, 2004.

DURANTE, M., E.; CANCELIER, A., C., L. Prevalência de internações por convulsões febris em serviço de pediatria no sul do país: análise dos fatores associados. Arquivos Catarinenses de Medicina, v. 36, n. 4, 2007.

FARIAS, E., B.; SILVA, L., W., C.; CUNHA, M., X., C. ABC AUTISMO: Um aplicativo móvel para auxiliar na alfabetização de crianças com autismo baseado no Programa TEACCH. X Simpósio Brasileiro de Sistemas de Informação, p. 458-469, 2014.

FENAPAES. Projeto Águia, Manual de Conceitos. Federação Nacional das Apaes, 2003.

FERREIRA, R., V.; COSTA, M., R.; DE MELO, D., C., S. Planejamento Familiar: gênero e significados. Textos \& Contextos, v. 13, n. 2, p. 387-397, 2014.

FIGUEIRÓ-FILHO, E., A.; LOPES, A., H., A.;SENEFONTE, F., R., DE A.; SOUZA JUNIOR, V., G., DE S.; BOTELHO, C., A.; DUARTE, G. Fenilcetonúria Materna: Relato de Caso. RBGO, v. 26, n. 10, 2004

FONTOURA, D., DA S. Mães Azuis: Trajetória de mães de autistas de Porto Alegre. Monografia Bacharelado em Ciências Sociais. Porto Alegre, 2015.

FORSAN, M., A. O uso indiscriminado de benzodiazepínicos: uma análise crítica das práticas de prescrição, dispensação e uso prolongado. Especialização em Atenção Básica. Campos Gerais/MG, 2010.

GARCIA, A., H., C.; VIVEIROS, M., M.; SCHWARTZMAN, J., S.; BRUNONI, D. Transtornos do espectro do autismo: avaliação e comorbidades em alunos de Barueri, São Paulo. Psicologia: teoria e prática, v. 18, n. 1, 2016.

GARCIA, J., M. Saúde Mental na Escola: O que os Educadores Devem Saber. Psico-USF, v. 21, n. 2, 2016.

GIL, A., C,. Como elaborar projetos de pesquisa. 4. ed. - São Paulo: Atlas, 2002.

GOMES, P., T., M.; LIMA, L., H., L.; BUENO, M., K., G.; ARAÚJO, L., A.; SOUZA, N., M. Autismo no Brasil, desafios familiares e estratégias de superação: revisão sistemática. Jornal de Pediatria, v. 91, n. 2, 2015. 
HADJKACEMA, I.; AYADI, H.; TURKI, M.; YAICHB, S.; KHEMEKHEMA, K.; WALHAA, A.; CHERIF, L.; MOALLAA, Y.; GHRIBI, F. Prenatal, perinatal and postnatal factors associated with autism spectrum disorder. Jornal de Pediatria, v. 92, n. 6, p. 595-601, 2016.

HALPERN, R.; FIGUEIRAS, A., C., M. Influências ambientais na saúde mental da criança. Jornal de Pediatria, v. 80, n. 2, 2004.

HERVÁS, A.; MARISTANY, M.; SALGADO, M.; SÁNCHEZ SANTOS, L. Los trastornos del espectro autista. Pediatría Integral, v. 16, n. 10, p. 780-94, 2012.

LEAL, M.; NAGATA, M.; CUNHA, N., DE M.; PAVANELLO, U.; FERREIRA, N., V., R. Terapia nutricional em crianças com transtorno do espectro autista. Cadernos da Escola de Saúde, v. 1, n. 13, 2015.

LEITE, R.; MEIRELLES, L., M., A.; MIHOMEM, D., B. Medicamentos usados no tratamento psicoterapêutico de crianças autistas em Teresina - PI. Boletim Informativo Geum, v. 6, n. 3, p. 91-97, 2015.

MACHADO, F., P.; LERNER, R.;NOVAES, B., C., DE A., C.; PALlADINO, R., R., R.; CUNHA, M., C. Questionário de indicadores clínicos de risco para o desenvolvimento infantil: avaliação da sensibilidade para transtornos do espectro do autismo. Audiology - Communication Research, v. 19, n. 4, 2014.

MAIA, F., A.; ALMEIDA, M., T., C.; OLIVEIRA, L., M., M., DE; OLIVEIRA, S., L., N.; SAEGER, V., S., DE A.; OLIVEIRA, V., S., D., DE; SILVEIRA, M., F. Importância do acolhimento de pais que tiveram diagnóstico do transtorno do espectro do autismo de um filho. Cadernos Saúde Coletiva, v. 24, n. 2, 2016.

MAIA FILHO, A., L., M.; NOGUEIRA, L., A., N., M.; SILVA, K., C., DE O.;SANTIAGO, R., F. A importância da família no cuidado da criança autista. Revista Saúde em Foco, v. 3, n. 1, 2016.

MANO, P. Abordagem e complicações medicamentosas na tontura. Revista HUPE, v. 14, n. 1, p. 37-41, 2015.

MARCHEZAN, J.; RIESGO, R., DOS S. Comorbidades dos trantonos do espectro autista. In: OHLWEILER, L.; ROTTA, N., T.; RIESGO, R., DOS S. Transtornos da Aprendizagem: Abordagem Neurobiológica e Multidisciplinar (e-book). Artmed Editora. 2015. Disponível em: <https://books.google.com.br/books?hl=pt$\mathrm{BR} \& \mathrm{lr}=\& \mathrm{id}=\mathrm{CdiGCgAAQBAJ} \& \mathrm{oi}=\mathrm{fnd} \& \mathrm{pg}=\mathrm{PA} 378 \& \mathrm{dq}=$ autismo/+retardo+mental $/+$ convuls\% $\mathrm{C} 3 \% \mathrm{~A} 3 \mathrm{o} \& \mathrm{ots}=\mathrm{y}$ 1bvgRE-up\&sig=QiIlMGpj8iB-Tp5mRLam7AzD-

$\mathrm{OA \# v}=$ onepage $\& \mathrm{q}=$ autismo $\% 2 \mathrm{~F} \% 20$ retardo $\% 20$ mental $\% 2 \mathrm{~F} \% 20$ convuls $\% \mathrm{C} 3 \% \mathrm{~A} 3 \mathrm{o} \& \mathrm{f}=$ false $>$, acessado em 01 de maio de 2017.

MATTOS, M., L.; PONDÉ, M., P.; CALMON, S. Alterações eletroencefalográficas em crianças com transtornos do espectro do autismo: revisão de prontuário. Psychiatry on Line Brasil, v. 18, n. 3, 2013.

MEDSCAPE. Risperidone. Disponível em: <http://reference.medscape.com/drug/risperdal-consta-risperidone342986\#4>, acessado em 01 de maio de 2017.

MEIMES, M., A.; SALDANHA, H., C.; BOSA, C., A. Adaptação Materna ao Transtorno do Espectro Autismo: Relações entre Crenças, Sentimentos e Fatores Psicossociais. PSICO, , v. 46, n. 4, pp. 412-422, 2015.

NIKOLOV, R.; JONKER, J.; SCAHILL, L. Autismo: tratamentos psicofarmacológicos e áreas de interesse para desenvolvimentos futuros. Revista Brasileira de Psiquiatria, v. 38, s. I, p. 39-46, 2006.

OLIVEIRA, F., C., DE A.; BARROS K., B., N., T.; SATURNO, R., DOS S.; LUZ, M., N., C.; VASCONCELOS, L., M., DE O. Perfil farmacoterapêutico de crianças autistas de uma clínica para reabilitação no estado do Ceará. Boletim Informativo Geum, v. 6, n. 3, p. 43-49, 2015.

OLIVEIRA, F., P., S., DE; FERREIRA, E., A., P. Adesão ao tratamento do hipotireoidismo congênito segundo relato de cuidadores. Psicologia: Reflexão e Crítica, v. 23, n. 1, 2010. 
OVIEDO, N.; MANUEL-APOLINAR, L.; CHESNAYEA, E., DE LA; GUERRA-ARAIZA, C. Aspectos genéticos y neuroendocrinos en el trastorno del espectro autista. Boletín Médico del Hospital Infantil de México, v. 72, n.1, p. 5-14, 2015.

PEREIRA, A.; PEGORARO, L., F., L.; CENDES, F. Autismo e epilepsia: modelos e mecanismos. Journal of Epilepsy and Clinical Neurophysiology, v. 18, n. 3, 2012.

PEREIRA, A.; RIESGO, R., S.; WAGNER, M., B. Autismo infantil: tradução e validação da Childhood Autism Rating Scale para uso no Brasil. Jornal de Pediatria, v. 84, n. 6, p. 487-494, 2008.

PEREIRA, M., E., C. Morel e a questão da degenerescência. Revista Latinoamericana de Psicopatologia Fundamental, v. 11, n. 3, 2008.

PINTO, R., N., M.;TORQUATO, I., M., B.; COLlE, N.; REICHERT, A., P., DA S.; SOUZA NETO, V., L., DE; SARAIVA, A., M. Autismo infantil: impacto do diagnóstico e repercussões nas relações familiares. Revista Gaúcha de Enfermagem, v. 37, n. 3, 2016.

RODRIGUES, M., N.; LIMA, S., R. Atividades motoras aquáticas na coordenação corporal de adolescentes com deficiência intelectual. Revista Brasileira de Ciências do Esporte, v. 36, n. 2, 2014.

SCARCELA, A., M., DE A.; MUNIZ, J., W., A.; CIRQUEIRA, J., Z. Investigação do uso indiscriminado de amoxicilina em crianças na faixa etária de 2 a 10 anos. Cenarium Pharmacêutico, n. 4, 2011.

SEMENSATO, M., R.; BOSA, C., A. Crenças parentais sobre o autismo e sua evolução no processo de comunicação diagnóstica. Pensando famílias, v. 18, n. 2, 2014.

SILVA, A., C., R., DA. Trabalho social com as famílias da pessoa com transtorno do espectro do autismo: uma análise na APAE de Presidente Prudente. Pós graduação, especialista em Trabalho Social com Famílias, 2015.

SILVA, L., C. DA; FRIGHETTO, A., M.; SANTOS, J., C., DOS. O Autismo e o Lúdico. Nativa, v. 1, n. $3,2013$.

SILVA, M.; MULICK, A. Diagnosticando o transtorno autista: aspectos fundamentais e considerações práticas. Psicologia Ciência e Profissão, Brasília, v. 29, n. 1, p. 116- 131, 2009.

SILVA, R., S.; CHAVES, E., F. Autismo, reações e consequências nas relações familiares. Encontro - Revista de Psicologia, v. 17, n. 26, 2014.

SOARES, T.; BRAGA, S., E., DE M. Relação da terapia de holding com a integração sensorial no autismo infantil. Revista Científica Interdisciplinar, v. 1, n. 2, p. 78-159, 2014.

SOUZA, V., M., DE A.; PEREIRA, A., M.; PALMINI, A.; PAGLIOLI NETO, E.; TORRES, C., M.; MARTÍNEZ, J., V.; COSTA, J., C., DA. Síndrome de West, autismo e displasia cortical temporal: resolução da epilepsia e melhora do autismo com cirurgia. Journal of Epilepsy and Clinical Neurophysiology, v. 14, n. 1, 2008.

TABAQUIM, M., DE L., M.; VIEIRA, R., G., DE S.; RAZERA, A., P., R.; CIASCA, S., M. Autoeficácia de cuidadores de crianças com o transtorno do espectro autista. Revista Psicopedagogia, v. 32, n. 99, 2015.

TEIXEIRA, R., M.; JUCÁ, V., J., DOS S. Caracterização dos usuários de um centro de atenção psicossocial infantojuvenil do município de Salvador (BA). Revista de Psicologia, v. 5, n. 2, p. 70-84, 2014.

VARELA, B.; MACHADO, P., G., B. Uma breve introdução sobre autismo. Cadernos da Escola de Educação e Humanidades, v.1 n.11: 25-39, 2016.

VASCONCELOS, M., M. Retardo Mental. Jornal Pediatria, v. 80, n. 2, 2004 
Id on Line Revista Multidisciplinar e de Psicoloqia

Id on Line Multidisciplinary and Psycology Journal

ZAMPIROLI, W., C.; SOUZA, V., M., P., DE. Autismo infantil. Uma breve discussão sobre a clínica e o tratamento. Pediatria Moderna, v. 48, n. 4, 2012.

ZANON, R., B.; BACKES, B.; BOSA, C., A. Identificação dos primeiros sintomas do autismo pelos pais. Psicologia: Teoria e Pesquisa, v. 30, n. 1, 2014.

\section{Como citar este artigo (Formato ABNT):}

FERNANDES, Lívia; PORTELA, Fernanda S.; MOREIRA, Pablo M.B.; FERNANDES, Mauro T. Perfil do uso de Medicamentos em Pacientes Autistas Acompanhados na APAE de um Município do Interior da Bahia. Id on Line Revista Multidisciplinar e de Psicologia, Maio de 2017, vol.11, n.35, p.301-316. ISSN: 1981-1179.

Recebido: 17.05.2017

Aceito: 25.05.2017 